8 Beyt BE, Ortbals DW, Santa Cruz DJ, Kobayashi GS, Eisen AZ, Medoff G. Cutaneous mycobacterioses. Analysis of 34 cases with a new classification of the disease. Medicin (Baltimore) 1980; 60: 95-108.

9 Breathnach A, Levell N, Munro C, Natarajan S, Pedler S. Cutaneous Mycobacterium kansasii infection: case report and review. Clin Infect Dis 1995; 20: 812-7.
10 Witzig RS, Franzblau SG. Susceptibility of Mycobacterium kansasii to ofloxacin, sparfloxacin, clarithromycin, azithromycin and fusidic acid: Antimicrob Agents Chemother 1993 $1997-9$.

\title{
Bacteraemia and intestinal obstruction in a 74-year-old man
}

\author{
GK Davis, MJ Brack, MT Hendrickse, DH Roberts
}

A 74-year-old man presented with a two-week history of fatigue, anorexia, upper abdominal pain and pyrexia. There was also a four-day history of constipation. On examination, he had a lowgrade pyrexia $\left(37.8^{\circ} \mathrm{C}\right)$ and an aortic ejection systolic murmur. There was no evidence of cardiac failure. Investigations showed a haemoglobin of $11.0 \mathrm{~g} / \mathrm{dl}$ with a mean corpuscular volume of $69 \mathrm{fl}$ and a significant neutrophilia. Chest X-ray showed cardiomegaly with clear lung fields. Transthoracic echocardiogram showed a calcified aortic valve without any significant stenosis or regurgitation. Twenty-four hours after admission he developed abdominal distension with radiological evidence of large and small bowel dilatation. Sigmoidoscopy was normal up to $20 \mathrm{~cm}$ and he settled on treatment with intravenous cefotaxime, metronidazole and nasogastric drainage. His antibiotic regimen was changed to high-dose intravenous penicillin when Streptococcus sanguis was isolated from his blood cultures. A barium enema examination could not be performed due to recurrent worsening left ventricular failure. A repeat transthoracic echocardiogram was of limited technical quality and showed a thickened, regurgitant aortic valve. He underwent transoesophageal echocardiography (figure) and had an emergency aortic valve replacement. Eight hours postoperatively, while being extubated, he suffered an unheralded cardiac arrest from which he could not be resuscitated.

\author{
Victoria Hospital, \\ Blackpool FY3 8NR, \\ UK \\ Regional Cardiac \\ Centre \\ GK Davis \\ MJ Brack \\ DH Roberts \\ Department of \\ Medicine \\ MT Hendrickse
}

Accepted 27 June 1996

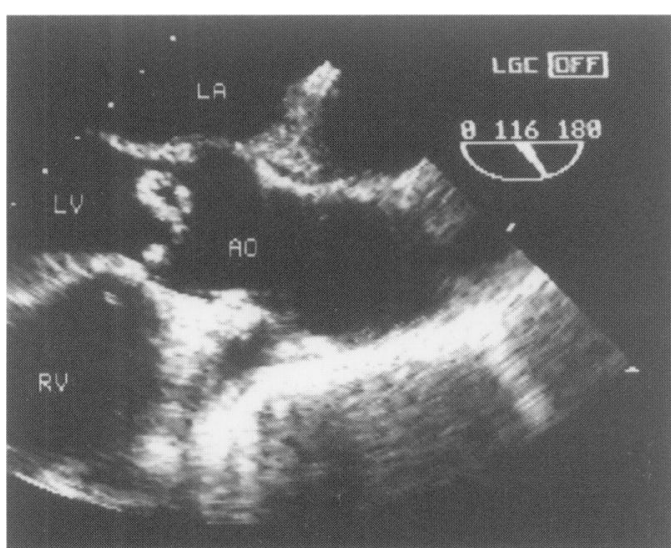

\section{Questions}

1 What is the most likely diagnosis?

2 What does the transoesophageal echocardiogram show?

3 What non-cardiac disorder might have been found at post-mortem?

Figure Transoesophageal echocardiography 


\begin{abstract}
Answers
QUESTION 1

The diagnosis is bacterial endocarditis due to Streptococcus sanguis.

QUESTION 2

The transoesophageal echocardiogram shows a large aortic cusp vegetation prolapsing into the left ventricular outflow tract. There was also severe aortic regurgitation on colour Doppler imaging (not illustrated).
\end{abstract}

\section{QUESTION 3}

At post-mortem examination the aortic prosthesis was intact and there was associated coronary atherosclerosis. In addition, there was a Duke's B annular rectosigmoid adenocarcinoma.

\section{Clinical features}

The association between bacterial endocarditis and adenocarcinoma of the colon was first described in 1951 in relation to $S$ faecalis bacteraemia. ${ }^{1}$ Similar observations have been made with $S$ bovis bacteraemia ${ }^{2}$ and bacteraemia due to streptococci of Lancefield's serogroup $\mathrm{D}$ ( $S$ equinus). ${ }^{3}$ The unexplained association between bacteraemia due to gut commensals and colonic cancer also extends to oral commensal organisms ${ }^{4}$ and hence the need to examine the lower gastrointestinal tract in patients with bacterial endocarditis due to oropharyngeal commensal organisms. Conversely, patients with known colonic malignancy who develop a pyrexia of unknown aetiology should have coexisting bacterial endocarditis excluded.

1 McCoy WC, Mason JM. Enterococcal endocarditis associated with carcinoma of the sigmoid. $\mathcal{F}$ Med Assoc State Ala 1951; 21: $162-6$.

2 Klein RS, Recco RA, Catalano MT, et al. Association of Streptococcus bovis with carcinoma of the colon. $N$ Engl ff Med 1977; 297: 800-2.

3 Gilon D, Moses A. Carcinoma of the colon presenting as Steptococcus equinus bacteremia. Am $\mathcal{F}$ Med 1989; 86: $135-6$.
Transoesophageal echocardiography in infective endocarditis

- more likely to be useful in elderly patients

- better sensitivity for detection of vegetations and better detection of abscess cavities than transthoracic echocardiography

- improves the identification of high-risk cases

\section{Investigation}

Transoesophageal echocardiography is an essential investigation in excluding bacterial endocarditis in patients with inadequate transthoracic echocardiograms. This is quite common in elderly patients who are more likely to have predisposing valvular conditions (such as calcified or degenerative valve lesions, as in this case) which decrease the sensitivity of the transthoracic echocardiogram to detect vegetations. The early use of transoesophageal echocardiogram in these patients has been shown by Werner $e t a \bar{T}$ to allow early diagnosis and initiation of medical and surgical therapy leading to a clinical outcome similar to that of young patients.

\section{Final diagnosis}

Streptococcus sanguis bacteraemia in association with bacterial endocarditis and colonic carcinoma.

Keywords: endocarditis, colonic cancer, Streptococcus sanguis bacteraemia, transoesophageal echocardiography

4 Siegert CEH, Overbosch D. Carcinoma of the colon presenting as Streptococcus sanguis bacteremia. Am f Gastroenterol 1995; 90: 1528-9.

5 Werner GS, Schulz R, Fuchs JB, et al. Infective endocarditis in the elderly in the era of transesophageal echocardiography: clinical features and prognosis compared with younger patients. Am f Med 1996; 100: 90-7. 